Accretion Phenomena and Related Outflows, IAU Colloquium 163

ASP Conference Series, Vol. 121, 1997

D.T. Wickramasinghe, L. Ferrario, and G.V. Bicknell, eds.

\title{
Wind Accretion vs Roche Lobe Overflow in HMXBs
}

\author{
John M. Blondin and Michael P. Owen \\ Dept. of Physics, North Carolina State University, Raleigh, NC 27695
}

\begin{abstract}
We present a series of time-dependent 2D and 3D numerical simulations illustrating the evolutionary sequence between high mass $\mathrm{X}$-ray binaries fed by wind accretion (where the primary star sits well within its critical tidal lobe) and those fed by Roche lobe overflow (where the primary star extends out to its tidal lobe). When the primary lies well within its critical surface we find negligible tidal mass loss enhancement, and a system that is characterized by wind accretion with the development of a photoionization zone around the compact object. As the surface of the primary nears the critical surface, we observe tidally enhanced mass loss via a thin tidal stream, resulting in higher accretion wake densities. For full RLOF we observe the development of a steady accretion disk characterized by a total shadowing of the X-rays in the orbital plane.
\end{abstract}

\section{Introduction}

High mass X-ray binaries consist of a binary system with an OB giant or supergiant primary star in close orbit with a collapsed companion, either a black hole or a neutron star. The intense X-ray luminosity of these systems is powered by mass accretion onto the collapse companion, which in turn is fueled by mass transfer from the primary star. Two possible explanations for the mass transfer are (1) Roche lobe overflow, and (2) gravitational capture of a stellar wind.

Perhaps the best discriminant between wind accretion and RLOF is the evolution of the spin period in X-ray pulsars. Although there is still much to be learned about the accretion of angular momentum in X-ray binaries (cf. Nelson), the observation of continued spin up in some HMXBs such as SMC X-1 and Cen $\mathrm{X}-3$ is strong evidence for disk accretion and hence RLOF, while the observation of a "random" walk in spin period in HMXBs such as Vela X-1 argues against the presence of an accretion disk, and hence for wind accretion. This classification is supported by the fact that systems with an observed continuous spin up also exhibit short pulse periods (if they have been spinning up continuously they must now be spinning fast), short orbital periods (the orbit must be close in order for the primary to contact the Roche lobe), and high X-ray luminosity.

Rather than being considered two separate classes, wind accretion and RLOF should be thought of as two extremes of a continuum of possibilities. Wind-accretion systems may have some tidal focusing of the wind even though the primary star does not fill its Roche lobe, and RLOF systems may still posses a strong stellar wind that may effect the accretion dynamics. Furthermore, the 
continuum can be thought of as a possible evolutionary sequence. In order for a wind-fed system to be detected, the compact companion must be in a relatively close orbit. If this is the case, further evolution of the system will likely bring the primary star into contact with its Roche lobe, driving the system into RLOF. Ultimately this evolution in mass transfer is expected to lead to a common envelope system.

The goal of the work described here is to investigate the properties of mass transfer in HMXBs as a function of the size of the primary star. Our approach is to use a series of two dimensional hydrodynamic simulations varying the size of the primary with respect to its Roche lobe. The numerical model is built upon the time-dependent hydrodynamic code VH-1. The 2D model computes the gas flow within the orbital plane, and includes the full potential of the rotating binary system and the radiation force of the primary star modeled in the Sobolev approximation. The influence of the X-rays in the model include X-ray heating, photoionization (disrupting the Sobolev force), and radiation pressure. Further details can be found in Blondin et al. (1990) and Owen \& Blondin (1997).

\section{2D Models}

The transition from wind accretion to RLOF is illustrated in Figure 1, where we plot the mass accretion rate onto the compact companion as a function of the RLOF parameter, $\lambda$, defined as the ratio of the effective potential at the surface of the primary to the Roche potential. At small values of $\lambda-1$ the primary is asymptotically approaching full RLOF, and the data points from the models are well fit by an exponential. Given that the surface of the primary is modeled as an isothermal (exponential) atmosphere, this result is simply stating that the mass loss rate from the primary (now dominated by the tidal stream) is proportional to the gas density at the inner Lagrangian point. At the other extreme, pure wind accretion, the mass loss rate from the primary has a shallow power law dependence on $\lambda-1$, originating from the dependence of a classical CAK wind on stellar radius. The resulting mass accretion rate is then fit reasonably well by a weak exponential, as shown in Figure 1.

The relatively sharp transition from wind-fed to RLOF in HMXBs is due to the small scale height in the atmosphere of the primary compared with the radius of the star. Once the upper atmosphere of the star comes in contact with the Roche surface, small changes in the stellar radius produce exponentially large changes in the mass transfer through the inner Lagrange point.

Another interesting feature of these simulations is the nature of the transitional case, where the mass loss rate from the primary is still dominated by the wind, but there is substantial focussing of this wind into a weak tidal stream. While the mass loss rate is nearly that of an isolated stellar wind, the mass accretion rate is significantly higher than in the wind accretion case. The large error bars in Figure 1 for this model signify extreme variations in the mass accretion rate (see Fig. 2) resulting from the dynamical interaction between the wind accretion bows hock and the weak tidal stream.

The temporal behavior of the accretion is significantly different, both between wind-fed and RLOF systems, but also compared to the transitional case. Figure 2 shows the X-ray light curve for a wind-fed, RLOF, and transitional 


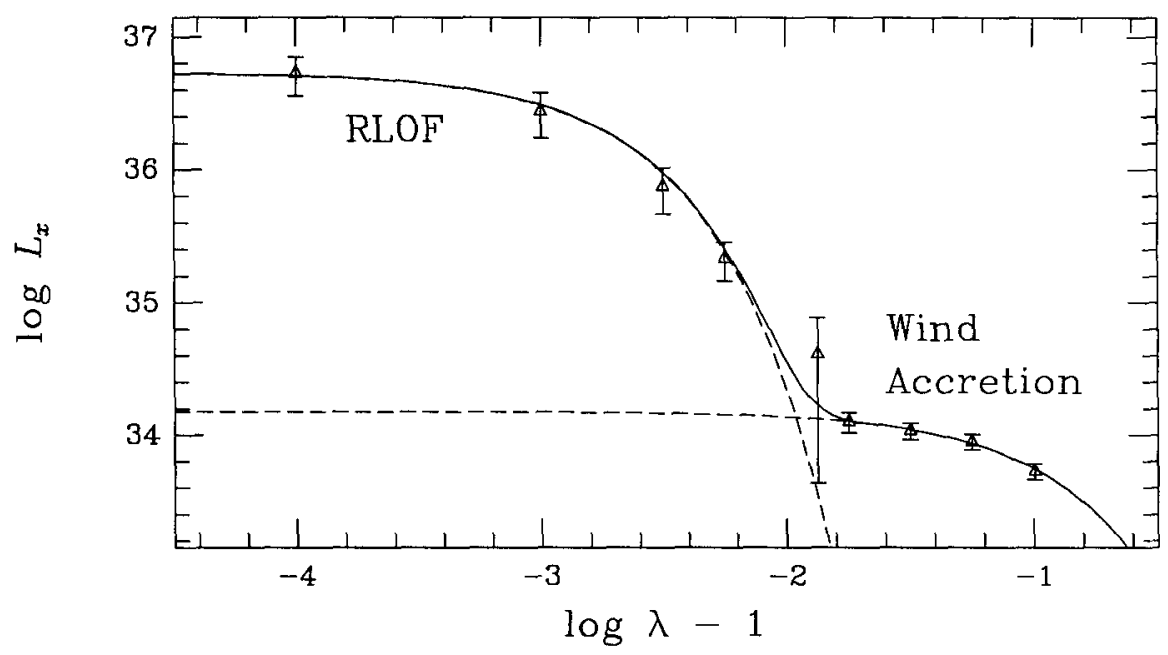

Figure 1. Accretion driven X-ray luminosity as a function of the RLOF parameter $\lambda$, defined in the text. The solid line is a fit using two exponential curves (dashed lines) corresponding to pure RLOF or wind accretion. The error bars on each model point represent the typical variation in $L_{x}$.

model. Note that to compute a mass accretion rate from a $2 \mathrm{D}$ model one must assume something about the neglected third dimension, and in particular what one assumes for wind-fed accretion is likely to be very different from disk accretion. We have opted to chose a height characteristic of disk accretion for all models, and for this reason the wind-fed models have a relatively low accretion driven luminosity. Furthermore, the wind accretion case has relatively poor numerical resolution.

Despite these caveats, Figure 2 illustrates what we believe to be real differences between these cases. For example, the disk case has significantly more power on long time scales (hours) than the wind case. The power spectrum from the disk model exhibits a roughly $\nu^{-1}$ power law with an additional broad peak at $\nu \sim 2 \times 10^{-3} \mathrm{~Hz}$. In contrast, the power spectrum from the wind model is almost flat at low frequencies. Another major difference is seen in the transitional case, which shows substantially larger variations than either wind or RLOF.

The results described above are useful for interpreting individual HMXBs and for understanding the evolution of these systems, but they are necessarily incomplete because mass transfer in binary systems is inherently three dimensional. The amplitude of the RLOF curve in Figure 1, and hence the location of the transition from RLOF to wind accretion will undoubtedly differ in three dimensions, but the essential conclusions should remain; the transition from wind-fed to RLOF in HMXBs is sharp, and on time scales of minutes to hours the temporal behavior of wind accretion, RLOF, and the transitional case is qualitatively different. 


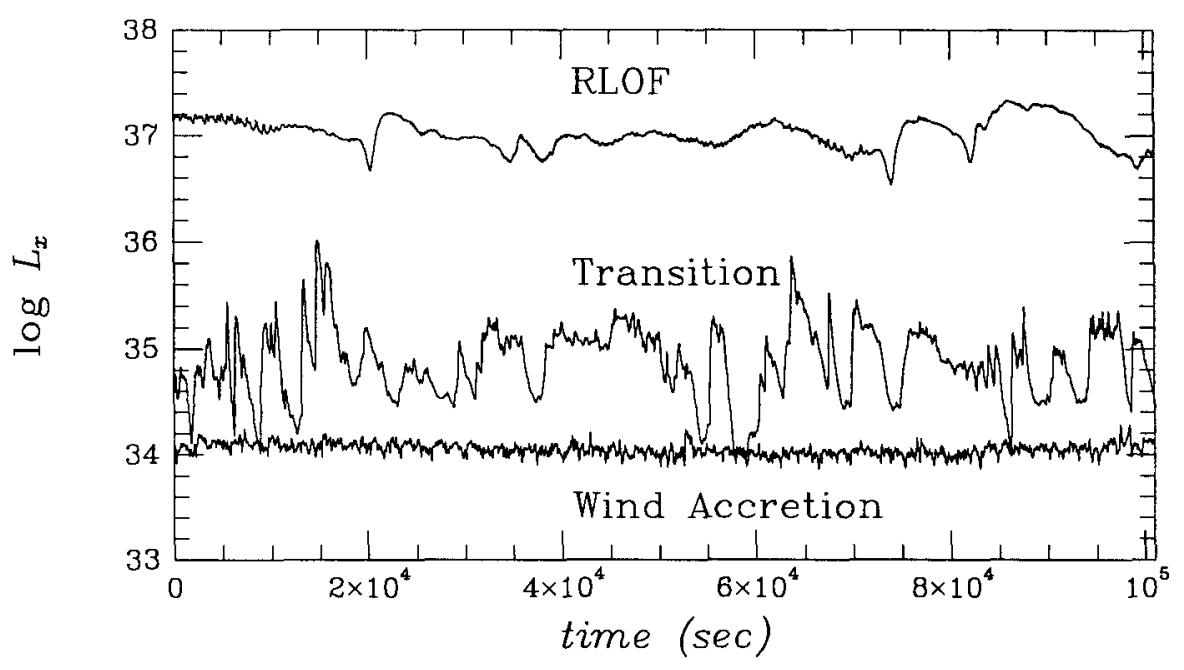

Figure 2. Accretion driven X-ray light curves from three different models corresponding to wind accretion, RLOF, and a transitional case.

We have begun to extend our simulations to $3 \mathrm{D}$, and while this work will be reported on in detail elsewhere, we include a brief description of a few preliminary results for the case of RLOF in Owen \& Blondin in these proceedings. We have also applied our 2D HMXB model to the elliptical orbit system GX 301-2 (Layton et al. 1997). These simulations suggest that tidal stripping is not occuring in GX 301-2, for while tidal stripping at periastron can lead to large $\mathrm{X}$-ray flares, such flares will occur at an orbital phase of $\sim 0.2$ after periastron.

\section{References}

Blondin, J. M., Kallman, T. R., Fryxell, B. A., \& Taam, R. E. 1990, ApJ, 356, 591

Layton, J. T., Blondin, J. M., \& Owen, M. P. 1996, NewA, submitted

Nelson, R. W. 1996, this volume

Owen, M. P. \& Blondin, J. M. 1997, in preparation

\section{Discussion}

P. Joss: Rather than Vela X-1 I would have chosen Cen X-3 as the "prototypical" wind-fed system. In the latter case, there is a great deal of evidence for a strong, roughly isotropic wind from the supergiant companion. Have you attempted to model the Cen X-3 system.

J. Blondin: We have not yet attempted a full 3D model of Cen X-3. In contrast to Vela $\mathrm{X}-1$, Cen X-3 shows a consistent spin up averaged over long timescales. 
This suggests that perhaps Cen $\mathrm{X}-3$ represents a transitional case between pure wind accretion and Roche lobe overflow.

$R$. Webbink: I am curious to hear your reaction to the BATSE results we saw yesterday - the seeming reversal of the sense of accretion from prograde to retrograde and back, at constant accretion rate. Have you found anything resembling this behaviour among your models?

$J$. Blondin: No. In wind accretion systems one can see changes in the sign of angular momentum accreted, as seen in Max Ruffert's simulations yesterday. However, the timescale for these changes is relatively short, whereas GX $1+4$ stays locked with one sign of angular momentum for years. I have not found any mechanism that can lock wind accretion into a retrograde disk. 